Honam Mathematical J. 35 (2013), No. 3, pp. 541-550

http://dx.doi.org/10.5831/HMJ.2013.35.3.541

\title{
NEW CARDINAL FUNCTIONS RELATED TO ALMOST CLOSED SETS
}

\author{
Myung Hyun $\mathrm{ChO}^{\dagger}$, Mi Ae Moon*, And Junhui Kim
}

\begin{abstract}
In this paper, we strengthen the properties of approximation by points (AP) and weak approximation by points (WAP) considered by A. Pultr and A. Tozzi in 1993 to define $\kappa$-AP and $\kappa$-WAP for an infinite cardinal $\kappa$. We also strengthen the properties of radial and pseudoradial to define $\kappa$-radial and $\kappa$-pseudoradial for an infinite cardinal $\kappa$. These allow us to consider new cardinal functions related to almost closed sets; AP-number, WAP-number, radial number, and pseudoradial number. We study their properties and show the relationships between them. We also provide some examples around $\kappa$-AP and $\kappa$-WAP which are closely connected with $\kappa$-radial and $\kappa$-pseudoradial.
\end{abstract}

\section{Introduction}

In this paper, all spaces are assumed to be Hausdorff. For any set $X$, the cardinality of $X$ is denoted by $|X| . \aleph_{0}$ is the first countable cardinal and $\aleph_{1}$ is the first uncountable cardinal. Undefined notions and terminologies can be found in [3].

A space $X$ is said to be $A P([2])$ if for every non-closed subset $A$ of $X$ and for every point $x \in \bar{A} \backslash A$, there exists a subset $F$ of $A$ such that $\bar{F}=F \cup\{x\}$. A space $X$ is said to be $W A P([2])$ if for every non-closed subset $A$ of $X$, there exist a point $x \in \bar{A} \backslash A$ and a subset $F$ of $A$ such that $\bar{F}=F \cup\{x\}$. Such a set $F$ is said to be almost closed. In particular, when the almost closed subset is countable in the definitions of AP and WAP, we say that the space $X$ is $A C P$ and $W A C P$ respectively ([4]).

A space $X$ is said to be radial if for every non-closed subset $A$ of $X$ and for every point $x \in \bar{A} \backslash A$, there exists a transfinite sequence

Received July 31, 2013. Accepted August 15, 2013.

2010 Mathematics Subject Classification. 54A25, 54A20, 54D55.

Key words and phrases. almost closed, $\kappa$-AP, $\kappa$-WAP, $\kappa$-radial, $\kappa$-pseudoradial.

${ }^{\dagger}$ This paper was supported by Wonkwang University in 2011.

${ }^{*}$ Corresponding author 
which converges to $x$. A space $X$ is said to be pseudoradial if for every non-closed subset $A$ of $X$, there exist a point $x \in \bar{A} \backslash A$ and a transfinite sequence which converges to $x$.

A space $X$ is called Fréchet-Urysohn if for any subset $A$ of $X$ and any $x \in \bar{A}$ there is a sequence in $A$ which converges to $x$. A space $X$ is called sequential if for any non-closed subset $A$ of $X$ there is a sequence in $A$ which converges to some $x \in \bar{A} \backslash A$. We say that a space $X$ has countable tightness, that is, $t(X) \leq \aleph_{0}$, if whenever $A \subset X$ and $x \in \bar{A}$, there exists a countable subset $B$ of $A$ such that $x \in \bar{B}$.

This paper is organized as follows. This section is to provide some notions and definitions which we will need afterwards. In section 2, we strengthen the properties of approximation by points (AP) and weak approximation by points (WAP) considered by A. Pultr and A. Tozzi ([5]) in 1993 to define $\kappa$-AP and $\kappa$-WAP for an infinite cardinal $\kappa$. We define new cardinal functions related to almost closed sets; AP-number and WAP-number. We study their properties and the relationships between them. We will be realized that these numbers can be considered only when the space $X$ is AP (or WAP, respectively). We also consider topological operations on $\kappa$-AP and $\kappa$-WAP spaces in the rest of this section. Section 3 is devoted to defining $\kappa$-radial and $\kappa$-pseudoradial which strengthen properties of radial and pseudoradial. We modify a

theorem by A. Bella and I. V. Yaschenko ([2]) that every pseudoradial AP space is radial to get a theorem [Theorem 3.4] which says that any $\kappa$-pseudoradial $\alpha$-AP space is $\kappa$-radial. This theorem implies that the radial number $r(X)$ is independent to the AP number $a p(X)$ when $X$ is pseudoradial. We prove a theorem [Theorem 3.6] that every countably compact $\kappa$-WAP space is $\kappa$-sequentially compact. AP and WAP spaces are closely related to radial and pseudoradial spaces, but it is interesting to see from Theorem 3.6 that the AP-number does not affect the the radial number in the class of countably compact spaces. We give an example of a Hausdorff space with $t(X)=\kappa$ and $r(X)=\kappa$ for any infinite cardinal $\kappa$ with $\operatorname{cf}(\kappa)=\kappa$. We also provide an example of a Hausdorff space which is $\kappa$-pseudoradial but not $\kappa$-radial.

\section{AP and WAP Numbers}

In this section, we study AP and WAP numbers related to almost closed sets. 
Definition 2.1. (1) A space $X$ is said to be $\kappa$-AP if for every nonclosed subset $A$ of $X$ and for every point $x \in \bar{A} \backslash A$, there exists a subset $F$ of $A$ such that $\bar{F}=F \cup\{x\}$ and $|F| \leq \kappa$.

(2) For any AP space $X$, we define the AP number $a p(X)$ as follows:

$$
a p(X)=\min \{\kappa: X \text { is } \kappa \text {-AP }\}+\aleph_{0} \text {. }
$$

Definition 2.2. (1) A space $X$ is said to be $\kappa$-WAP if for every nonclosed subset $A$ of $X$, there exist a point $x \in \bar{A} \backslash A$ and a subset $F$ of $A$ such that $\bar{F}=F \cup\{x\}$ and $|F| \leq \kappa$.

(2) For any WAP space $X$, we define the WAP number wap $(X)$ as follows:

$$
\operatorname{wap}(X)=\min \{\kappa: X \text { is } \kappa \text {-WAP }\}+\aleph_{0} .
$$

We give general properties without proof because each can be obtained by definitions.

Proposition 2.3. The following hold for any space $X$ :

(1) $X$ is AP if and only if $X$ is $\kappa$-AP for some cardinal $\kappa$;

(2) $X$ is WAP if and only if $X$ is $\kappa$-WAP for some cardinal $\kappa$;

(3) $X$ is $A C P$ if and only if $a p(X)=\aleph_{0}$;

(4) $X$ is WACP if and only if $\operatorname{wap}(X)=\aleph_{0}$;

(5) every Fréchet-Urysohn space is $\aleph_{0}-A P$;

(6) every sequential space is $\aleph_{0}-W A P$;

(7) every $\kappa$-AP space is $\kappa$-WAP;

(8) every $\kappa$-AP space is $A P$; and

(9) every $\kappa$-WAP space is WAP.

Proposition 2.4. For every $\kappa$-WAP space $X$, the tightness of $X$ is less than or equal to $\kappa$, that is, $t(X) \leq \operatorname{wap}(X)$.

Proof. Let $X$ be a $\kappa$-WAP space and $A$ be a non-closed subset of $X$. Since $X$ is $\kappa$-WAP, there exist a point $p \in \bar{A} \backslash A$ and a subset $F$ of $A$ such that $\bar{F}=F \cup\{p\}$ and $|F| \leq \kappa$. Therefore $t(X) \leq \kappa$.

W. C. Hong has considered AP and WAP properties of Examples $2.5,2.6$, and 2.7 below (see [4] for details). These examples will be investigated again in the point of view of cardinal functions.

Example 2.5. Let $X=\{z\} \cup \mathbb{R}$, where $\mathbb{R}$ is the set of all real numbers. We define a topology $\tau$ on $X$ by

(i) $\{x\} \in \tau$ for all $x \in \mathbb{R}$; and

(ii) $z \in U \in \tau$ if and only if $\mathbb{R} \backslash U$ is countable.

Then $X$ is Hausdorff. From the fact that $z$ is the unique non-isolated point of $X$, we obtain that every non-closed subset is almost closed. 
Since every countable subset of $\mathbb{R}$ is closed, every non-closed subset must be uncountable. Therefore $a p(X)=w a p(X)=t(X)=\aleph_{1}$.

Recall that $\tau_{1} \vee \tau_{2}$ is the smallest topology on $X$ generated by two topologies $\tau_{1}$ and $\tau_{2}$ on $X$.

Example 2.6. Let $\tau_{1}$ be the usual topology on $\mathbb{R}$ and $\tau_{2}$ be the countable complement topology on $\mathbb{R}$. Let $\tau=\tau_{1} \vee \tau_{2}$. Then $(\mathbb{R}, \tau)$ is not AP. Therefore $(\mathbb{R}, \tau)$ is not $\kappa$-AP for all cardinal $\kappa$.

The next example is a WAP space $X$ which is not AP. In particular, the WAP number $\operatorname{wap}(X)$ is equal to $\aleph_{0}$.

Example 2.7. Let $X=(\omega \times \omega) \cup \omega \cup\{z\}$ be the space with a base defined by

(i) each point of $\omega \times \omega$ is isolated;

(ii) a basic open neighbourhood of $n \in \omega$ is the form of

$$
V_{k}(n)=\{n\} \cup\{<m, n>: m \geq k\} ; \quad \text { and }
$$

(iii) a basic open neighbourhood of $z$ is the form of

$$
W_{p}(z)=\{z\} \cup \bigcup\left\{V_{0}(n) \backslash F_{n}: n \geq p\right\},
$$

where $p \in \omega$ and $F_{n}$ is a finite subset of $V_{0}(n)$.

Then $X$ is Hausdorff and sequential. Hence $\operatorname{wap}(X)=\aleph_{0}$ by Proposition 2.3(6). Let $A=\omega \times \omega$. Note that for each subset $F$ of $A$ with $z \in \bar{F}$, $\omega \cap \bar{F}$ is infinite. Hence there is no subset $F$ of $A$ such that $\bar{F}=F \cup\{z\}$. Therefore $a p(X) \neq \kappa$ for any infinite cardinal $\kappa$.

Example 2.8 below is the classical example which explains that there exists a space which is not $\kappa$-WAP for any infinite cardinal $\kappa$.

Example 2.8. Let $X$ be an infinite set with the finite complement topology. Suppose that $X$ is $\kappa$-WAP for some infinite cardinal $\kappa$. Choose a subset $A$ of $X$ such that both $A$ and $X \backslash A$ are infinite. Then $A$ is not closed in $X$. Since $X$ is $\kappa$-WAP, there exist a point $p \in \bar{A} \backslash A$ and a subset $F$ of $A$ such that $\bar{F}=F \cup\{p\}$ and $|F| \leq \kappa$. Since $F$ is infinite, $\bar{F}=X$. So we can choose a point $q \in \bar{F} \backslash F$ such that $p \neq q$, which is a contradiction. Therefore $X$ is not $\kappa$-WAP for any cardinal $\kappa$.

As we have seen in the above examples, the cardinal number $a p(X)$ (or $\operatorname{wap}(X)$ ) can be considered when the space $X$ is AP (or WAP, respectively). Moreover, we have the following when $X$ is a AP space.

Theorem 2.9. Let $X$ be a AP space with $\kappa$-WAP. Then $X$ is $\kappa$-AP. In other words, $a p(X)=w a p(X)$ for any AP space $X$. 
Proof. Let $A$ be a non-closed subset of $X$ and let $p \in \bar{A} \backslash A$. Since $X$ is AP, there exists a subset $B$ of $A$ such that $\bar{B}=B \cup\{p\}$. Then we can find a subset $F$ of $B$ such that $\bar{F}=F \cup\{p\}$ and $|F| \leq \kappa$ from the facts that $X$ is $\kappa$-WAP and $\bar{B} \backslash B=\{p\}$. Therefore $X$ is $\kappa$-AP.

By summarizing the above results, we obtain some implications as in Diagram 1. Notice that, for any infinite cardinal $\kappa, \kappa$-AP and $\kappa$-WAP are equivalent properties when a space is AP by Theorem 2.9.

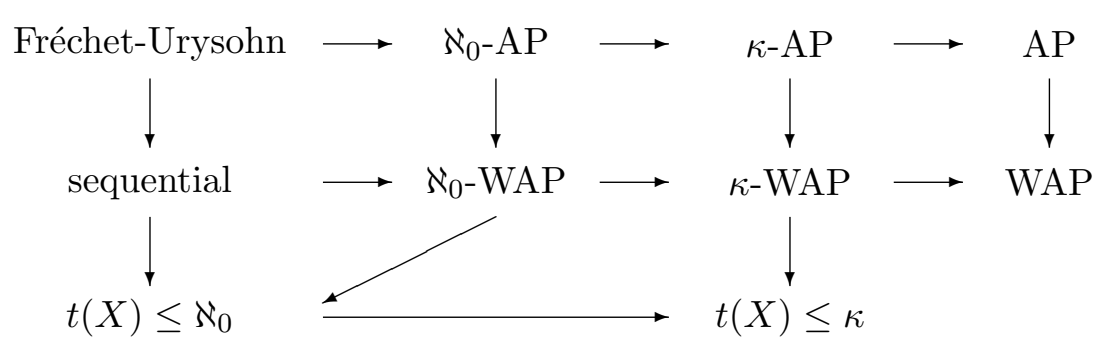

Diagram 1. $\kappa$ is an infinite cardinal

In the rest of this section, we consider topological operations on $\kappa$-AP and $\kappa$-WAP spaces.

Theorem 2.10. The following hold for any space:

(1) any subspace $Y$ of a $\kappa$-AP space $X$ is $\kappa-A P$, that is, $a p(Y) \leq a p(X) ;$

(2) any closed subspace $Y$ of a $\kappa$-WAP space $X$ is $\kappa$-WAP, that is, $\operatorname{wap}(Y) \leq \operatorname{wap}(X) ;$ and

(3) any open subspace $Y$ of a $\kappa$-WAP space $X$ is $\kappa$-WAP, that is, $\operatorname{wap}(Y) \leq \operatorname{wap}(X)$.

Proof. (1) Suppose that $Y$ is a subspace of a $\kappa$-AP space $X$. Let $A$ be a non-closed subset of $Y$ and let $p \in \bar{A}^{Y} \backslash A$. Then $p \in \bar{A}^{X} \backslash A$. Since $X$ is $\kappa$-AP, there exists a subset $F$ of $A$ such that $\bar{F}^{X}=F \cup\{p\}$ and $|F| \leq \kappa$. Then $\bar{F}^{Y}=\bar{F}^{X} \cap Y=(F \cup\{p\}) \cap Y=F \cup\{p\}$. Therefore $Y$ is $\kappa$-AP.

(2) Suppose that $Y$ is a closed subspace of a $\kappa$-WAP space $X$. Let $A$ be a non-closed subset of $Y$. Then $A$ is a non-closed subset of $X$. Since $X$ is $\kappa$-WAP, there exist a point $p \in \bar{A}^{X} \backslash A$ and a subset $F$ of $A$ such that $\bar{F}^{X}=F \cup\{p\}$ and $|F| \leq \kappa$. It follows from $\bar{Y}^{X}=Y$ that $p \in \bar{F}^{X} \subset \bar{Y}^{X}=Y$. Thus $\bar{F}^{Y}=F \cup\{p\}$. Therefore $Y$ is $\kappa$-WAP.

(3) Suppose that $Y$ is an open subspace of a $\kappa$-WAP space $X$. Let $A$ be a non-closed subset of $Y$. Then $A \cup(X \backslash Y)$ is not closed in $X$. Since 
$X$ is $\kappa$-WAP, there exist a point $p \in \overline{A \cup(X \backslash Y)}^{X} \backslash[A \cup(X \backslash Y)]$ and a subset $F$ of $A \cup(X \backslash Y)$ such that $\bar{F}^{X}=F \cup\{p\}$ ad $|F| \leq \kappa$. Then $p \in \overline{A \cup(X \backslash Y)}{ }^{X}=\bar{A}^{X} \cup \overline{(X \backslash Y)}^{X}=\bar{A}^{X} \cup(X \backslash Y)$. Also it follows from $p \notin A \cup(X \backslash Y)$ that $p \notin A$ and $p \in Y$. Hence $p \in \bar{A}^{Y} \backslash A$ and $\bar{F}^{Y}=(F \cup\{p\}) \cap Y=F \cup\{p\}$. Therefore $Y$ is $\kappa$-WAP.

Theorem 2.11. The following hold for any space:

(1) any closed continuous image of a $\kappa$-AP space is $\kappa$-AP;

(2) any closed continuous image of a $\kappa$-WAP space is $\kappa$-WAP; and

(3) a quotient image of an $\aleph_{0}-A P$ space is not necessarily $\aleph_{0}-A P$.

Proof. (1) Suppose that $X$ is a $\kappa$-AP space and $f: X \rightarrow Y$ is a closed continuous onto map. Let $A$ be a non-closed subset of $Y$ and $p \in \bar{A} \backslash A$. Denote $B=f^{-1}(A)$. Then $f^{-1}(p) \cap \bar{B} \neq \emptyset$. Indeed, suppose $f^{-1}(p) \cap \bar{B}=\emptyset$. Then $p \notin f(\bar{B})$. Since $f$ is continuous and closed, $f(\bar{B})=\overline{f(B)}=\bar{A}$. So $p \notin \bar{A}$, a contradiction.

So $B$ is not closed in $X$ (because $\left.f^{-1}(p) \cap B=\emptyset\right)$. Since $X$ is $\kappa$-AP, for all $y \in f^{-1}(p) \cap \bar{B}$, there is a subset $C$ of $B$ such that $\bar{C}=C \cup\{y\}$ and $|C| \leq \kappa$. Take $D=f(C)$. Then $D \subseteq f(B)=A,|D| \leq \kappa$, and $\bar{D}=\overline{f(C)}=f(\bar{C})=f(C \cup\{y\})=f(C) \cup\{f(y)\}=D \cup\{p\}$. Therefore $Y$ is $\kappa$-AP.

(2) It is similar with (1).

(3) V. V. Tkachuk and I. V. Yaschenko gave a non-AP space which is a quotient image of a metric space $([1,6])$. Since every metric space is first countable, it is $\aleph_{0}$-AP. Hence a quotient image of an $\aleph_{0}$-AP space is not necessarily $\aleph_{0}$-AP.

\section{Radial and Pseudoradial Numbers}

To study radial and pseudoradial spaces in the point of view of convergence of transfinite sequences for some given length $\kappa$, we introduce some definitions as follows:

Definition 3.1. (1) A space $X$ is called $\kappa$-radial if for every nonclosed subset $A$ of $X$ and for every point $p \in \bar{A} \backslash A$, there exists a transfinite sequence $\left\{x_{\alpha} \in A: \alpha<\kappa\right\}$ which converges to $p$.

(2) For any radial space $X$, we define the radial number $r(X)$ as follows:

$$
r(X)=\min \{\kappa: X \text { is } \kappa \text {-radial }\}+\aleph_{0} .
$$


Definition 3.2. (1) A space $X$ is called $\kappa$-pseudoradial if for every non-closed subset $A$ of $X$, there exist a point $p \in \bar{A} \backslash A$ and a transfinite sequence $\left\{x_{\alpha} \in A: \alpha<\kappa\right\}$ which converges to $p$.

(2) For any pseudoradial space $X$, we define the pseudoradial number $\operatorname{pr}(X)$ as follows:

$$
\operatorname{pr}(X)=\min \{\kappa: X \text { is } \kappa \text {-pseudoradial }\}+\aleph_{0} .
$$

One can prove easily the following properties by definitions.

Proposition 3.3. The following hold for any space $X$ :

(1) $X$ is radial if and only if $X$ is $\kappa$-radial for some $\kappa$;

(2) $X$ is pseudoradial if and only if $X$ is $\kappa$-pseudoradial for some $\kappa$;

(3) $X$ is $\aleph_{0}$-radial if and only if $X$ is Fréchet-Urysohn;

(4) $X$ is $\aleph_{0}$-pseudoradial if and only if $X$ is sequential;

(5) every $\kappa$-radial space $X$ is $\kappa$-pseudoradial;

(6) every $\kappa$-radial space $X$ is $\kappa$-AP; and

(7) every $\kappa$-pseudoradial space $X$ is $\kappa$-WAP,

Theorem 3.4 below is a modification of the statement which have been proved by A. Bella and I. V. Yaschenko ([2]): every pseudoradial AP space is radial. This statement implies that $r(X)$ is independent to $a p(X)$ when $X$ is pseudoradial.

Theorem 3.4. Let $\kappa$ and $\alpha$ be any infinite cardinals. Then any $\kappa$-pseudoradial $\alpha$-AP space is $\kappa$-radial.

Proof. Suppose that $X$ is a $\kappa$-pseudoradial $\alpha$-AP space. Let $A$ be a non-closed subset of $X$ and let $p \in \bar{A} \backslash A$. Since $X$ is $\alpha$-AP, we can find a subset $F$ of $A$ such that $\bar{F}=F \cup\{p\}$ and $|F| \leq \alpha$. Since $F$ is not closed in $X$, there exists a transfinite sequence $S \subset F$ with $|S| \leq \kappa$ which converges to some point $x$ outside $F$. By the almost closedness of $F$, the point $x$ must be equal to $p$. Therefore $X$ is $\kappa$-radial.

Corollary 3.5. For any $A P$ space $X, \kappa$-radiality and $\kappa$-pseudoradiality are equivalent. Hence $\operatorname{pr}(X)=r(X)$.

We introduce a definition which is an extension of sequential compactness: a space $X$ is called $\kappa$-sequentially compact if every transfinite sequence has a convergent transfinite subsequence with length $\leq \kappa$.

Theorem 3.6. Every countably compact $\kappa$-WAP space is $\kappa$-sequentially compact. 
Proof. Let $X$ be a countably compact $\kappa$-WAP space and let $f: \alpha \rightarrow$ $X$ be a one-to-one transfinite sequence in $X$ for each $\alpha \geq \kappa$. By the countable compactness of $X$, we can find a cluster point $p$ of $f(\alpha)$. Since $A=f(\alpha) \backslash\{p\}$ is not closed in $X$ and $X$ is $\kappa$-WAP, there exist a subset $B \subset A$ and $q \in X \backslash A$ such that $\bar{B}=B \cup\{q\}$ and $|B| \leq \kappa$. Choose a minimal family of open sets $\left\{U_{\beta}: \beta<\kappa\right\}$ in the subspace $\bar{B}$ with $\bigcap_{\beta<\kappa} \bar{U}_{\beta}=\{q\}$. Then we have

$$
\bigcap_{\gamma<\beta} \bar{U}_{\gamma} \backslash\{q\} \neq \emptyset \quad \text { for all } \beta<\kappa .
$$

Now, we take a point $p_{\beta} \in \bigcap_{\gamma<\beta} \bar{U}_{\gamma} \backslash\{q\}$. Since $\bar{B}$ is countably compact, the transfinite sequence $\left\{p_{\beta}: \beta<\kappa\right\}$ converges to $q$. By construction, this sequence is a subsequence of $f$. Therefore $X$ is $\kappa$-sequentially compact.

Remark 3.7. V. V. Tkachuk and I. V. Yaschenko ([6]) have proved that every countably compact AP space is Fréchet-Urysohn. This statement can be expressed in our terminology as "every countably compact $\kappa$-AP space is $\aleph_{0}$-radial". This means that the number ap $(X)$ does not affect the number $r(X)$ in the class of countably compact spaces.

We omit the proof of Theorem 3.8 below because it can be shown by a similar way with Theorem 2.9 .

Theorem 3.8. Let $X$ be a radial space with $\kappa$-pseudoradial. Then $X$ is $\kappa$-radial. In other words, $r(X)=\operatorname{pr}(X)$ for any radial space $X$.

By summarizing the above results, we obtain some implications as in Diagram 2. Notice that, for any infinite cardinal $\kappa, \kappa$-radial and $\kappa$ pseudoradial are equivalent properties when a space is radial by Theorem 3.8 .

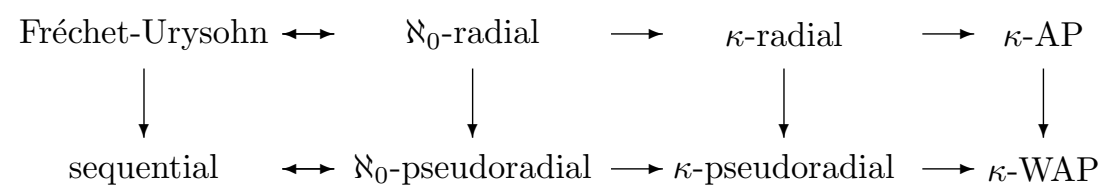

Diagram 2. $\kappa$ is an infinite cardinal

Finally, we conclude this section by introducing some examples.

Recall that $\operatorname{cf}(\kappa)$ is the cofinality of a cardinal $\kappa$. The following example explains the existence of a Hausdorff space $X$ with $r(X)=\kappa$ for any infinite cardinal $\kappa$ with $\operatorname{cf}(\kappa)=\kappa$. 
Example 3.9. For any infinite cardinal $\kappa$ with $\operatorname{cf}(\kappa)=\kappa$, let $D$ be any set with $|D|=\kappa$ and fix $p \notin D$. Now we topologize $X=D \cup\{p\}$ as follows:

(i) every point of $D$ is isolated; and

(ii) if $p \in U \subset X$, then $U$ is open if and only if $|X \backslash U|<\kappa$.

Then one can easily see that $X$ is a Hausdorff space with $t(X)=\kappa$. We now show that $r(X)=\kappa$. Since every subset $F$ of $X$ with $|F|<\kappa$ is closed in $X$, it is enough to show that $X$ is $\kappa$-radial. Let $A$ be a non-closed subset of $X$. Then $|A|=\kappa$ and $p \in \bar{A} \backslash A$. We enumerate $A=\left\{x_{\alpha}: \alpha<\kappa\right\}$. Let $U$ be an open neighbourhood of $p$. Then $|X \backslash U|<\kappa$. Since $\operatorname{cf}(\kappa)=\kappa$ and $|A \backslash U|<\kappa$, we can choose $\beta<\kappa$ such that if $\alpha>\beta$, then $x_{\alpha} \in A \cap U$. Therefore $X$ is $\kappa$-radial.

Example 3.10. For an infinite cardinal $\kappa$ with $\operatorname{cf}(\kappa)=\kappa$, let $X=$ $(\kappa \times \kappa) \cup \kappa \cup\{z\}$ be the space with a base defined by

(i) each point of $\kappa \times \kappa$ is isolated;

(ii) a basic open neighbourhood of $\beta \in \kappa$ is the form of

$$
V_{\gamma}(\beta)=\{\beta\} \cup\{\langle\alpha, \beta>: \alpha \geq \gamma\} ; \quad \text { and }
$$

(iii) a basic open neighbourhood of $z$ is the form of

$$
W_{\delta}(z)=\{z\} \cup \bigcup\left\{V_{0}(\beta) \backslash F_{\beta}: \beta \geq \delta\right\},
$$

where $\beta \in \kappa$ and $F_{\beta}$ is a subset of $V_{0}(\beta)$ such that $\left|F_{\beta}\right|<\kappa$.

Then $X$ is Hausdorff. And one can easily show that $\operatorname{pr}(X)=\kappa$. Let $A=\kappa \times \kappa$. Since, for each subset $F$ of $A$ with $z \in \bar{F},|\kappa \cap \bar{F}|=\kappa$, there is no subset $F$ of $A$ such that $\bar{F}=F \cup\{z\}$. Therefore $X$ is not $\kappa$-AP and hence $X$ is not $\kappa$-radial.

Acknowledgement. The authors would like to thank the referee for valuable suggestions and careful reading.

\section{References}

[1] A. V. Arhangel'skii, Bicompact sets and the topology of spaces, Soviet Mathematics, Doklady, 4 (1963), 561-564.

[2] A. Bella and I. V. Yaschenko, On AP and WAP spaces, Comment. Math. Univ. Carolin. 40(3) (1999), 531-536.

[3] R. Engelking, General Topology, Revised and completed edition, Heldermann Verlag, Berlin, 1989.

[4] W. C. Hong, Generalized Fréchet-Urysohn Spaces, J. Korean Math. Soc. 44(2) (2007), 261-273. 
[5] A. Pultr and A. Tozzi, Equationally closed subframes and representation of quotient spaces, Cahiers Topologie Géom. Différentielle Catég. 34(3) (1993), 167183.

[6] V. V. Tkachuk and I. V. Yaschenko, Almost closed sets and topologies they determine, Comment. Math. Univ. Carolin. 42(2) (2001), 393-403.

Myung Hyun Cho

Department of Mathematics Education, Wonkwang University, Iksan 570-749, Korea.

E-mail: mhcho@wonkwang.ac.kr

Mi Ae Moon

Division of Mathematics \& Informational Statistics, Wonkwang University,

Iksan 570-749, Korea.

E-mail: moonmae@wonkwang.ac.kr

Junhui Kim

Department of Mathematics Education, Wonkwang University, Iksan 570-749, Korea.

E-mail: junhikim@wonkwang.ac.kr 